\title{
Baseband Transmission on Power Line Channels with LDPC Coset Codes
}

\author{
Raju Hormis $^{\dagger}$, Inaki Berenguer ${ }^{\S \dagger}$, and Xiaodong Wang ${ }^{\dagger}$ \\ ${ }^{\dagger}$ Columbia University, New York, NY \\ $\S$ University of Cambridge, Cambridge, UK
}

\begin{abstract}
High rate transmission over power lines imposes stringent requirements in coding and equalization, the chief impairments being severe signal attenuation and impulsive noise. Yet, transmission requirements over this medium have reached several Mbit/sec to compete with other types of access. In this paper, we propose a novel PAMbased coded modulation scheme that is well suited to meeting such constraints. The proposed scheme combines Low Density Parity Check (LDPC) codes and maximum-distance separable block codes to achieve high spectral efficiency, low decoding complexity, and a high degree of immunity to impulse noise. To achieve better immunity to burst and impulse noise, a novel interleaving scheme is proposed. To achieve good performance in the presence of inter-symbol interference, the proposed coset-coding is combined with Tomlinson-Harashima precoding and spectral shaping at the transmitter.
\end{abstract}

\section{INTRODUCTION}

The power-line channel presents severe constraints for communications, such as signal attenuation (insertion loss) over long cables, impulse noise, and inter-symbol interference. In particular, impulse noise is a severe impairment, and occurs in the form of timevarying periodic noise synchronized to the line frequency, periodic but asynchronous noise caused by switching power supplies, and asynchronous noise caused by random switching transients in the network (cf. [3], [17]). In this paper, we propose a simple PAMbased coset coding scheme to overcome these impairments. For brevity, we focus on static or slowly time-varying channels. The modulation scheme offers high spectral efficiency, immunity to multiple impulse noise sources, good coding gains, but yet, requires low complexity overall. The idea of coding with cosets within a lattice framework was first generalized in [6]. An important result, proven in [7], [16], is that coset codes can achieve the sphere bound - channel capacity without shaping - with simple 1-dimensional lattices, and with two or three levels of coset partitioning. Motivated by these ideas, the scheme proposed here for power-line channels is based on a 3-level coset decomposition with different codes at each layer of the decomposition. Viewing the bottom layer as a Gaussian channel at low SNR, the scheme relies on (3,6)-regular low-rate LDPC codes of 1000-2000 bits [10] for steep BER reduction. Meanwhile, the middle layer is treated as a binary symmetric channel (BSC) that is coded with hard-decision random and burst error-correcting cyclic codes. In particular, a high-rate Reed-Solomon (RS) code is applied here to protect against random and phased-burst errors. By virtue of its large intra-coset distance, the top layer of the decomposition can be viewed as a BSC channel that is only vulnerable to burst noise. Both RS codes and single-burst-error correcting codes from [11] are investigated.

In work related to LDPC-based coded modulation, a cosetcoding scheme was proposed in [5] for DMT modulation over digital subscriber lines. The authors demonstrated that coset codes can be constructed with relatively short LDPC binary codes, thus keeping latency to a minimum. Independently of this work, one of us proposed a coset-coding scheme for twisted-pair transmission at $10 \mathrm{Gbit} / \mathrm{sec}$ [13], with LDPC codes on the order of $2000 \mathrm{bits}$.
Here, performance at a BER of $10^{-12}$ was $2.5 \mathrm{~dB}$ from the sphere-bound. A DMT-based LDPC coset-coding scheme was also proposed recently in [1] for ISI-constrained channels; the scheme makes use of RS component codes at high layers due to their low complexity and their well-known construction at high rates. However, the motivation of our proposal is broader, as we view the coset decomposition with different noise characteristics at each layer. The partition between modulation, coding and equalization is also different from what is proposed here.

To extend the burst error-correction ability of the proposed coding schemes, interleaving is applied. Uniform interleaving is widely used in concatenated coding where, for example, a softdecision code like trellis-coded modulation (TCM) is concatented with an RS code, separated by a byte-interleaver [14]. The RS code in such schemes corrects burst-errors that are left uncorrected by the Viterbi decoder. However, the scheme proposed in this paper differs in the sense that interleavers are used at each layer of a coset decomposition independently, each tailored to different properties of the component codes and noise at different layers. Simulation results show good performance with sufficient interleaver depth.

The remainder of the paper is organized as follows. In Section II, the power-line system model is outlined, along with details of the channel and noise. We also motivate the proposed transmission scheme in this section. Section III elaborates on the design of the proposed coset code in Gaussian noise as a first step to designing for the power-line channel. In Section IV, the scheme is augmented to handle synchronous and asynchronous impulse noise. Simulation results are also presented. Section V concludes the paper.

\section{SySTEM DESCRIPTIONS}

In this section, we review the transmission model and channel conditions for power-line communications, focusing on last-mile access over low-voltage lines.

\section{A. Channel Transfer Function}

A major drawback of power-lines is that the cable follows a bus topology, rather than a point-to-point connection. Each powerline connecting each house or main to the bus (branch) can have a different terminating impedance. Terminations (e.g., open mains or connected appliances) represent a complex impedance causing reflections (return loss), and consequently, a multi-path channel at the receiver. Moreover, longer paths experience higher attenuations since the signals travel longer distances. Thus, the frequency response of the PLC multi-path channel $H(f)$ can be approximated by a sum of $N$ paths [18]. The sum accounts for multi-path propagation and frequency-selective fading, viz.,

$$
H(f)=\sum_{i=1}^{N} \underbrace{g_{i}}_{\text {weighting }} \underbrace{e^{-\left(a_{0}+a_{1} f^{k}\right) d_{i}}}_{\text {attenuation }} \underbrace{e^{-j 2 \pi f \frac{d_{i}}{v_{p}}}}_{\text {delay }},
$$

where $g_{i}$ represents a weighting factor along path $i$ with distance $d_{i} ; a_{0}, a_{1}$ are attenuating parameters; $k$ is the exponent of the 
attenuation, usually in the interval 0.2 to 1 . The last term represents the propagation delay, with $v_{p}$ denoting the velocity of propagation. Typical values of $a_{0}, a_{1}$, and $k$ are given in [18].

In this paper, we consider values of $g_{i}, d_{i}, N, a_{0}, a_{1}$, and $k$ that represent a typical reference channel ("channel 3 ") for lastmile access, whose parameters are based on real measurements in Germany [18]. Channel 3 represents a hostile channel consisting of a $210 \mathrm{~m}$ line with 8 branches, and hence multiples sources of reflected signal power. The impulse response lasts on the order of $10 \mu \mathrm{s}$. An important characteristic that we depend on is the symmetry of the transfer function (cf. [2]), which holds true when the terminating impedances of the transceivers are identical at both ends of the link. This property permits transmitter-side techniques, as will be shown in Section II-C.

\section{B. Additive Noise}

A comprehensive analysis in [17] characterized the noise sources that afflict power lines. The authors showed that the various noise sources can be classified broadly into the following categories:

a) Colored Gaussian noise.

b) Narrow-band sinusoidal noise (EMI) that originates from commercial AM, FM and ham radio sources.

c) Periodic impulsive noise that is synchronous to the mains (i.e. every $\mathrm{AC}$ cycle) originated by transients in appliances connected to the power lines.

d) Periodic impulse noise that is asynchronous to the mains, caused by switching power supplies.

e) Asynchronous and aperiodic impulsive noise usually caused by random switching transients.

In this paper, we consider all noise sources mentioned above, except b). While narrow-band EMI is a significant constraint in practical schemes, for this paper, we assume that the narrowband interference can be mitigated with a frequency notch, using a combination of spectral shaping at the transmitter and noisewhitening matched filter at the receiver. Meanwhile, the Gaussian noise is assumed to be strongly colored, with higher energy at low frequencies.

1) Synchronous Impulse Noise: It has been measured that a high percentage of the impulsive noise occurs periodically and synchronously to the mains. In general, the impulsive noise consists of a collection of damped sinusoids [3], with higher content in the low frequencies. The periodic impulses can be modelled as a collection of $I_{s}$ damped sinusoids

$n_{s}(t)=\sum_{i=1}^{I_{s}} A_{i} \sin \left(2 \pi f_{i}\left(t-t_{a r r, s}\right)+\alpha_{i}\right) e^{-\frac{t-t_{a r r, s}}{\tau_{i}}} \Pi\left(\frac{t-t_{a r r, s}}{t_{w, s}}\right)$

where $f_{i}$ is the "pseudo-frequency" of the sinusoid, and $\alpha_{i}$ the phase, of the $i$-th damped sinusoid. $\Pi(t)$ is defined as a square pulse of duration $t_{w, s} \mathrm{sec}$, with constant amplitude in the interval $0<t \leq 1$ and zero elsewhere. $t_{a r r, s}$ is the periodic arrival time, and $A_{i}$ denotes the amplitude of the $i$-th sinusoid. We assume $A_{i} \sim \mathcal{N}\left(0, G_{i} \sigma_{v}^{2}\right), i=1 \ldots I_{s}$, where $G_{i}$ represents the increase over the variance of Gaussian background noise $\sigma_{v}^{2}$, and can range from $20-30 \mathrm{~dB}$. The gain $G_{i}$ of sinusoids at higher pseudo-frequencies is selected to match the typical low frequency content observed in impulsive noise measurements, usually below $1 \mathrm{MHz}$. The term $\tau_{i}$ denotes the damping factor. Meanwhile, the pulse amplitude equals the standard deviation of the background noise. In [17], impulses of approximately $t_{w, s}=50 \mu \mathrm{s}$ have been measured, and this value is used in the simulations. In [3], pseudofrequencies were characterized from $500 \mathrm{KHz}$ to $3 \mathrm{MHz}$. In this paper, we consider 3 component sinusoids $\left(I_{s}=3\right)$, with pseudofrequencies of $300 \mathrm{KHz}, 2 \mathrm{MHz}$, and $3.5 \mathrm{MHz}$.
2) Asynchronous Impulse Noise: The combination of all impulsive noise sources that are asynchronous to the main frequency can be modelled as a sum of damped sinusoids as in (2), but where arrival time $t_{a r r, a}$ is modelled as a random variable [17]. The asynchronous bursts are usually caused by switching transients. Let $t_{I A T, a}=t_{a r r, a}^{(p)}-t_{a r r, a}^{(p-1)}$ denote the inter-arrival time between consecutive bursts of asynchronous impulse noise, viz., burst $p$ and $p-1$. Then, as discovered in [17], $t_{I A T, a}$ can be modelled with an exponential distribution. In the simulations, we select $t_{I A T, a}$ to be exponentially distributed with mean of $100 \mathrm{~ms}$. We assume the impulse width $t_{w, a}$ to be constant, approximately $100 \mu \mathrm{s}$. However, the amplitudes of the sinusoids $A_{i}, i=1 \ldots I_{a}$ remain Gaussian distributed, as in Section II-B.1.

\section{Transmission Model}

We employ a simple baseband PAM-based scheme in this paper, with an emphasis on static or slowly time-varying channels. In stationary Gaussian noise and under the condition of zero excessbandwidth, a PAM-based scheme - when combined with ideal DFE, spectral shaping at the transmitter, and noise-whitening matched filter - is asymptotically capacity-achieving at high SNR (cf. [8]). On the other hand, the impulse noise statistics are timevariant on the order of a few micro-seconds [17]; this makes it difficult to compute even the capacity of such a channel. To simplify the design of a transmitter in impulse noise, we take a decidedly sub-optimal approach. First, the shaping transmit-filter, equalizer and matched-filter are computed with well-known methods for an ISI-constrained Gaussian channel. In summary, these techniques present a flat AWGN channel to a channel decoder, which greatly simplifies the design of a coding scheme (Section III). The code is then augmented to protect against non-stationary impulse noise (Section IV).

The scheme is depicted in Fig. 1. $S(f)$ represents a spectralshaping filter, designed to achieve the optimal water-filling spectrum for the power-line channel $H(f)$. The well-known minimum mean-squared error (MMSE) DFE is used to mitigate ISI. Let $B(z)$ denote the feedback filter of the DFE that cancels post-cursor ISI, assuming perfect decision-feedback. Due to the difficulty of combining DFE's with block codes, the proposed scheme makes use of the well-known Tomlinson-Harashima (TH) precoding [8]. This entails using $B(z)$ in a feedback loop at the transmitter to mitigate post-cursor ISI a priori, as shown in Fig. 1. The symmetry of the channel transfer function proven in [2] permits precoding. The TH-precoding induces a small transmitter power-penalty; for an $M$-PAM constellation, the penalty has been shown to be $\frac{M^{2}}{M^{2}-1}$, which is asymptotically negligible for large constellations. However, the TH-precoder also causes shaping loss by up to $\pi e / 6 \approx 1.53 \mathrm{~dB}$. To avoid the shaping loss, a practical alternative is Laroia precoding, which is asymptotically capacity-achieving at high SNR's with large constellations [8]. A receiver filter, $W(z)$ in Fig. 1, denotes the noise-whitening matched filter of the DFE. This filter also mitigates pre-cursor ISI. We are now left with almostGaussian residual ISI and whitened Gaussian noise at the input to the channel decoder. This motivates our approach of designing the coding scheme for the AWGN channel first.

\section{Coding In Gaussian NoISE}

In this section, we propose a coset-coding technique that combines both bandwidth-efficiency and good performance in the presence of Gaussian noise.

\section{A. Code Structure}

We refer the reader to a comprehensive treatment of lattices and coset codes in [6]. The modulation scheme of this paper uses PAM constellations, which can be viewed as sub-sets of lattices. The 

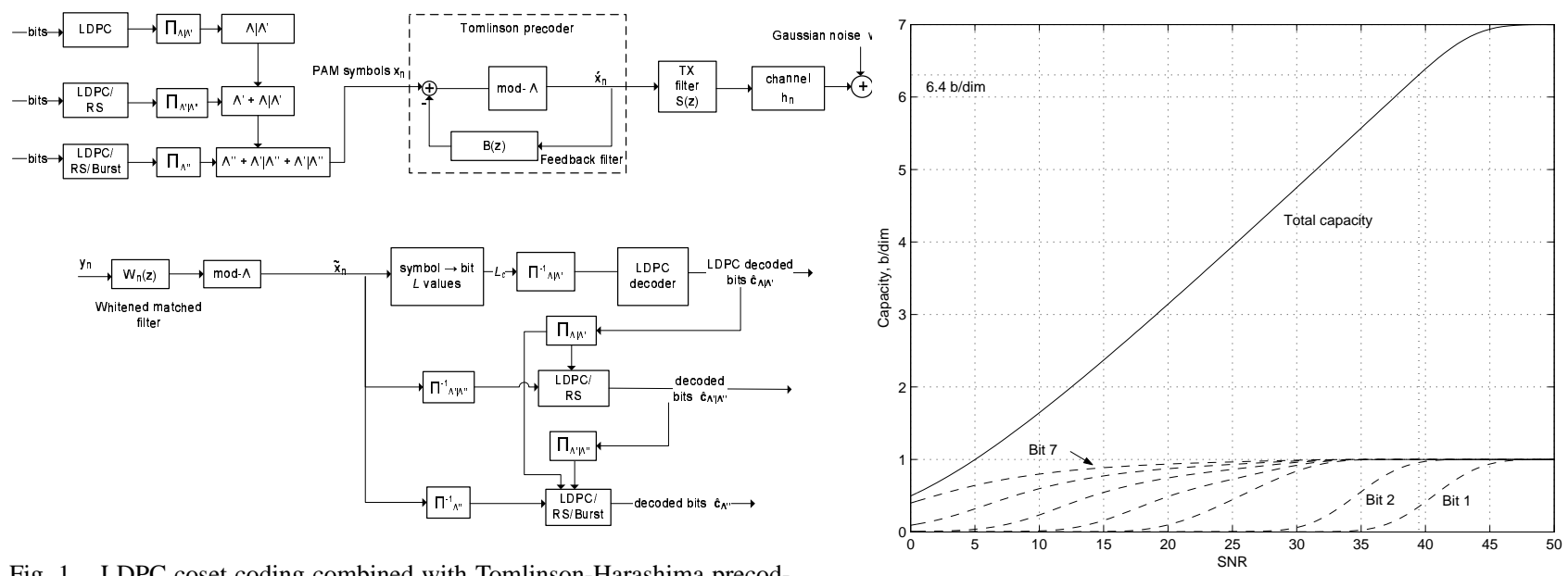

Fig. 1. LDPC coset coding combined with Tomlinson-Harashima precoding.

Fig. 2. Capacity analysis of 3-level coset partition for 128-PAM, as in [16].

PAM constellation can be viewed as a finite set of points belonging to a translate of an $N$-dimensional lattice $\Lambda$ and bounded by a rectangular shaping region $\mathcal{S}$. The constellation can be expressed as $(\Lambda+\Omega) \cap \mathcal{S}$, where $\Omega \in \mathbb{R}^{N}$ is a translation vector. $\Omega$ is selected to center a constellation symmetrically around the origin. Consider a sub-lattice $\Lambda^{\prime}$ of $\Lambda$. A coset of $\Lambda^{\prime}$ can be defined as a translation of $\Lambda^{\prime}$ by $\lambda$, such that

$$
\Lambda^{\prime}+\lambda \triangleq\left\{\boldsymbol{x}=\boldsymbol{u}+\lambda \mid \boldsymbol{x} \in \Lambda, \quad \boldsymbol{u} \in \Lambda^{\prime}, \quad \lambda \in\left[\Lambda \mid \Lambda^{\prime}\right]\right\} .
$$

$\left[\Lambda \mid \Lambda^{\prime}\right]$ represents the set of translates that satisfies (3). The sublattice $\Lambda^{\prime}$ is said to induce a partition, $\Lambda \mid \Lambda^{\prime}$, of the infinite lattice $\Lambda$. The coset partitions $\Lambda^{\prime}\left|\Lambda^{\prime \prime}, \Lambda^{\prime \prime}\right| \Lambda^{\prime \prime \prime}, \cdots$ of a multi-level partition chain can be defined similarly.

Consider a 3-level lattice partition chain $\Lambda\left|\Lambda^{\prime}\right| \Lambda^{\prime \prime}$. Let $\boldsymbol{G}_{\Lambda \mid \Lambda^{\prime}}$ and $\boldsymbol{G}_{\Lambda^{\prime} \mid \Lambda^{\prime \prime}}$ denote generator matrices of block codes that respectively generate codewords $\boldsymbol{c}_{\Lambda \mid \Lambda^{\prime}}$ and $\boldsymbol{c}_{\Lambda^{\prime} \mid \Lambda^{\prime \prime}}$ over alphabets $\left[\Lambda \mid \Lambda^{\prime}\right]$ and $\left[\Lambda^{\prime} \mid \Lambda^{\prime \prime}\right]$. A third matrix $\boldsymbol{G}_{\Lambda^{\prime \prime} \mid \Lambda^{\prime \prime \prime}}$ generates codewords $c_{\Lambda^{\prime \prime} \mid \Lambda^{\prime \prime \prime}}$ that selects $m$ points from a sub-set $\left\{\left(\Lambda^{\prime \prime}+\Omega\right) \cap \mathcal{S}\right\}$. Now, a coset-code $\boldsymbol{L}$ can be defined as a set of codewords selected such that

$$
\boldsymbol{L} \triangleq\left\{\boldsymbol{x}=\boldsymbol{c}_{\Lambda \mid \Lambda^{\prime}}+\boldsymbol{c}_{\Lambda^{\prime} \mid \Lambda^{\prime \prime}}+\boldsymbol{c}_{\Lambda^{\prime \prime} \mid \Lambda^{\prime \prime \prime}} \mid \boldsymbol{x} \in \Lambda^{m}\right\} .
$$

The examples in this paper use a 3-level coset partition over $\mathbb{Z}$. The bits mapped on $\left\{\left(\Lambda^{\prime \prime}+\Omega\right) \cap \mathcal{S}\right\}$ are left uncoded in Gaussian noise, but coded for impulse noise. If the rates of each component code of the coset decomposition are $R_{\Lambda \mid \Lambda^{\prime}}, R_{\Lambda^{\prime} \mid \Lambda^{\prime \prime}}$ and $R_{\Lambda^{\prime \prime}}$, it is easy to see that the coding rate of $\boldsymbol{L}$ is given by

$$
\begin{gathered}
R(\boldsymbol{L})=\frac{1}{N}\left[R_{\Lambda \mid \Lambda^{\prime}} \log _{2}|\Lambda| \Lambda^{\prime}\left|+R_{\Lambda^{\prime} \mid \Lambda^{\prime \prime}} \log _{2}\right| \Lambda^{\prime}\left|\Lambda^{\prime \prime}\right|+\right. \\
\left.R_{\Lambda^{\prime \prime}} \log _{2}\left|\left(\Lambda^{\prime \prime}+\Omega\right) \cap \mathcal{S}\right|\right] \mathrm{b} / \mathrm{dim}
\end{gathered}
$$

\section{B. Code Construction in Gaussian Noise}

In this section, we discuss the code construction, coset decomposition, and choice of coding rate for a slowly time-varying powerline channel.

Capacity Considerations and Rate Allocation: Let $\mathrm{C}(\boldsymbol{L})$ denote the capacity of a coset code $\boldsymbol{L}$ over a lattice partition $\Lambda\left|\Lambda^{\prime}\right| \Lambda^{\prime \prime}$, and let $C_{\Lambda \mid \Lambda^{\prime}}, C_{\Lambda^{\prime} \mid \Lambda^{\prime \prime}}$ and $C_{\Lambda^{\prime \prime}}$ denote the capacities of each layer of the coset decomposition. A key result proved in [16] is that $\mathrm{C}(\boldsymbol{L})$ can be achieved by any combination of coding rates, provided $R_{\Lambda \mid \Lambda^{\prime}}+R_{\Lambda^{\prime} \mid \Lambda^{\prime \prime}}+R_{\Lambda^{\prime \prime}}=\mathrm{C}(\boldsymbol{L})$. In particular, apportioning $\mathrm{C}(\boldsymbol{L})$ by matching coding rate to partition capacity, i.e.,

$$
R_{\Lambda \mid \Lambda^{\prime}}:=\mathrm{C}_{\Lambda \mid \Lambda^{\prime}}, R_{\Lambda^{\prime} \mid \Lambda^{\prime \prime}}:=\mathrm{C}_{\Lambda^{\prime} \mid \Lambda^{\prime \prime}}, R_{\Lambda^{\prime \prime}}:=\mathrm{C}_{\Lambda^{\prime \prime}},
$$

has an important benefit in terms of reducing complexity. This choice of rate-allocation allows soft-decision multi-stage decoding to be used without loss of optimality, assuming capacity-achieving component codes are used. This rate allocation strategy is used in the paper, but for simplicity, we use hard-decision decoding.

To compute the rate allocation, consider a nominal transmit power of $0 \mathrm{dBmW}$. The water-filling capacity analysis in Section II$\mathrm{C}$ revealed a capacity of approximately $49.7 \mathrm{Mbit} / \mathrm{sec}$ for "channel 3" over a frequency band $0 \leq f \leq 3.85 \mathrm{MHz}$. We implement a zero excess-bandwidth PAM-based scheme, operating at $7.7 \mathrm{MHz}$, leading to transmission of $6.44 \mathrm{~b} / \mathrm{symbol}$. On the other hand, the capacity of a multi-level decomposition of 128-PAM in Gaussian noise is shown in Fig. 2. The figure shows that a capacity of 6.44 $\mathrm{b} / \mathrm{dim}$ can be achieved at a minimum SNR of about $38.9 \mathrm{~dB}$ at the input to a coset code demodulator. For slowly time-varying channels, the rate-allocation must be re-computed periodically with coordination from the receiver.

With this approach in mind, the analysis shows that rate $R_{\Lambda \mid \Lambda^{\prime}} \approx$ 0.5 is optimal to code the $\Lambda \mid \Lambda^{\prime}$ partition, while $R_{\Lambda^{\prime} \mid \Lambda^{\prime \prime}} \approx 0.9$ is optimal to code $\Lambda^{\prime} \mid \Lambda^{\prime \prime}$. The analysis also shows that the bits mapped onto $\left\{\left(\Lambda^{\prime \prime}+\Omega\right) \cap \mathcal{S}\right\}$ can be transmitted at full rate in Gaussian noise. This justifies the choice of a simple 3-level coset partition.

Selection of Component Codes: To obtain a steep reduction in BER, we select $\boldsymbol{G}_{\Lambda \mid \Lambda^{\prime}}$ as short $(3,6)$-regular LDPC codes from [10], which are known to have good performance at low rates. The codes have length 1000-2000 bits which results in low complexity. For $G_{\Lambda^{\prime} \mid \Lambda^{\prime \prime}}$, we consider two choices: a rate- 0.9 regular LDPC code from [4], and a rate-0.9 Reed-Solomon (RS) code. It can be shown that a relatively weak algebraic code $\boldsymbol{G}_{\Lambda^{\prime} \mid \Lambda^{\prime \prime}}$ is sufficient for the $\Lambda^{\prime} \mid \Lambda^{\prime \prime}$ partition, under hard-decision multi-stage decoding.

\section{Discussion of Simulation Results}

Based on the code construction discussed previously, two coset codes are investigated here: one making use of LDPC codes, and the other a combination of LDPC and RS codes. Both schemes use hard-decision multi-stage decoding. By the capacity analysis of Fig. 2, both schemes use the same $(3,6)$-regular rate- 0.5 regular LDPC code from [10] for $\boldsymbol{G}_{\Lambda \mid \Lambda^{\prime}}$. Furthermore, the top layer is left uncoded in both schemes. However, the schemes differ in the choice of encoder matrix $G_{\Lambda^{\prime} \mid \Lambda^{\prime \prime}}$ : one is a rate-0.94 regular LDPC code from [4], while the other is a binary expansion of an RS code of same rate. Codes around 1000-2000 bits in length are used in all cases. To our knowledge, the family of LDPC codes in [4] exhibit the best performance among regular high-rate codes. 


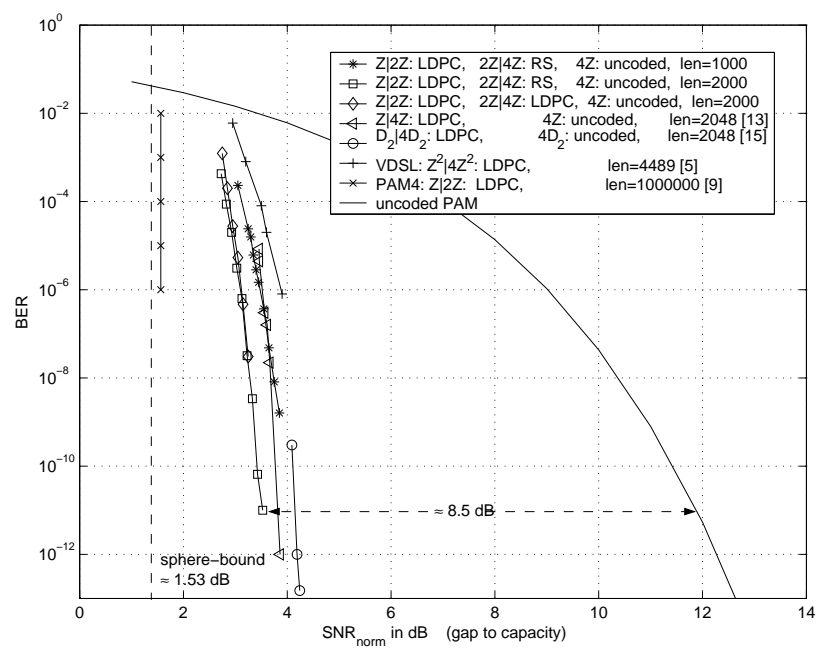

Fig. 3. Comparison of various LDPC-coded modulation schemes

The performance of the schemes is shown in Fig. 3, relative to gap-to-capacity or normalized SNR. The scheme combining LDPC and RS codes lies within $2 \mathrm{~dB}$ of the sphere-bound $-3.5 \mathrm{~dB}$ of Shannon-capacity - at a BER of $10^{-11}$, measured with simulations over $2.5 \times 10^{12}$ bits. There is almost no difference in performance to the LDPC-only example proposed above for BER's measured up to $10^{-7}$. A second example - with a $(3,6)$-regular code of 1000 bits for $\boldsymbol{G}_{\Lambda \mid \Lambda^{\prime}}$ - shows similar performance to recent proposals for 10G-Base-T Ethernet [13], [15] which uses (6,32)-regular codes of 2048 bits. However, the complexity of the schemes proposed in this paper is far lower due to the small degree of the nodes in $\boldsymbol{G}_{\Lambda \mid \Lambda^{\prime}}$.

The results can be compared to other LDPC-based coded modulation schemes. In [9] for example, 2-level coding schemes over $\mathbb{Z} \mid 2 \mathbb{Z}$ with a total rate of $1 \mathrm{~b} /$ sym were analyzed. The schemes considered irregular and quasi-regular LDPC codes for $\boldsymbol{G}_{\Lambda \mid \Lambda^{\prime}}$ and $G_{\Lambda^{\prime} \mid \Lambda^{\prime \prime}}$ with length $10^{6}$ bits each. Here, a gap to the spherebound of 0.2-0.3 dB was observed. In [5], the authors investigated LDPC coset-coding over DMT modulation on $\mathbb{Z}^{2} \mid 4 \mathbb{Z}^{2}$ with QAM constellations for VDSL applications, with component codes on the order of 2000-4000 bits. The scheme is about $1 \mathrm{~dB}$ away in performance from proposals in this paper. As mentioned in Section ??, the authors of [1] proposed a combination of LDPC and RS codes for coset-coding over DMT on $\mathbb{Z}^{2}\left|2 \mathbb{Z}^{2}\right| 4 \mathbb{Z}^{2}$. In the presence of AWGN, the gap to Shannon capacity of the scheme in [1] was found to be about $2.3 \mathrm{~dB}(0.8-1.2 \mathrm{~dB}$ gap to the spherebound). The justification for RS codes was to reduce complexity and to ease code selection, which is only part of the rationale of our paper. Meanwhile, to handle different coding rates on each sub-channel, a concatenation of LDPC and various repeat-codes are used on the $\Lambda \mid \Lambda^{\prime}$ partition.

\section{Coding in Gaussian And Impulse Noise}

In this section, we investigate LDPC-based coset coding under the simultaneous constraints of colored Gaussian noise and impulse noise. Both synchronous and asynchronous impulse noise models from Section II-B.2 are considered, and different coding schemes are compared.

\section{A. Error-Correction Schemes across $\Lambda\left|\Lambda^{\prime}\right| \Lambda^{\prime \prime}$}

Coding on $\Lambda \mid \Lambda^{\prime}$ : This layer is affected by both Gaussian noise and burst impulse noise. However, the coding scheme here makes use of the same LDPC codes $\boldsymbol{G}_{\Lambda \mid \Lambda^{\prime}}$ used in the AWGN case of Section III-C. The goal is to obtain a steep reduction in BER. The LDPC construction is based on [10], and at low coding rates (only), seems to perform well even in the presence of impulse noise.
Coding on $\Lambda^{\prime} \mid \Lambda^{\prime \prime}$ : The capacity analysis of Fig. 2 in Gaussian noise showed that the $\Lambda^{\prime} \mid \Lambda^{\prime \prime}$ partition could be coded at a high rate due to the smaller impact of Gaussian noise at this layer. However, the effect of impulse noise is to cause bursts of errors, either in the form of a single-burst or as multiple phased-bursts. Viewing $\Lambda^{\prime} \mid \Lambda^{\prime \prime}$ as a binary symmetric channel (BSC) for simplicity, we are now interested in a code $G_{\Lambda^{\prime} \mid \Lambda^{\prime \prime}}$ that can correct both random and burst errors efficiently, with little loss in rate. The burst-correction efficiency, $\eta$, of an $(n, k)$ code can be defined [12] by the amount of redundancy required to correct all error bursts of length $l$-bits or less, viz.,

$$
\eta=\frac{2 l}{n-k} .
$$

A code that can correct all bursts of length $l$-bits or less with an efficiency of $\eta=1$ is said to achieve the Reiger bound. Though not optimum, we rely on the random and phased-burst error-correcting properties of RS cyclic codes for $\Lambda^{\prime} \mid \Lambda^{\prime \prime}$. A binary expansion of a $t$-error correcting $(n, k)$ Reed-Solomon code over $\mathrm{GF}\left(2^{q}\right)$ can correct:

- Any combination of $t$ or fewer random bit errors.

- A single burst of length $l=(t-1) q+1$ bits, or less.

- Any combination of $\frac{t}{1+\lfloor(l+q-2) / q\rfloor}$ separate bursts of length $l$, in bits [12].

These properties follow from the fact that a $G F\left(2^{q}\right)$ RS code operates on $q$-bit symbols. Notice that, as $q \rightarrow \infty, \eta=1$. Hence, RS codes are asymptotically optimum.

Coding on $\Lambda^{\prime \prime}$ : The errors of this layer are dominated by long single-burst errors or multiple phased-bursts. There are virtually no errors due to Gaussian noise. Viewing $\Lambda^{\prime \prime}$ as a BSC channel, we investigate RS codes, as well as simple cyclic codes over $G F(2)$ optimized for single-burst error-correction [11]. For comparison, LDPC codes are also considered. The main attraction of singleburst error-correcting codes is their low complexity (lengths on the order of 100-200 bits) coupled with high efficiency $\eta$. In particular, a $(195,182)$ code from [11] is considered, with $\eta \approx 0.77$.

\section{B. Multi-Level Interleaving}

To extend the burst error-correction ability of the coding schemes above, uniform interleavers are used at each layer of a coset decomposition. The proposed scheme is depicted in Fig. 1 and consists of uniform interleavers $\Pi_{\Lambda}, \Pi_{\Lambda^{\prime}}$, and $\Pi_{\Lambda^{\prime \prime}}$ respectively. For the combination of LDPC and cyclic codes, the scheme can be summarized as follows:

1. Uniform Bit-Interleaving of LDPC Code Bits with $\Pi_{\Lambda \mid \Lambda^{\prime}}$ : Let $d_{H}\left(G_{\Lambda}\right)$ denote the minimum Hamming distance of the LDPC code, and let $\Psi_{\boldsymbol{L}}$ denote the interleaver depth of coset code $\boldsymbol{L}$. Then, with optimal decoding, a well-known result from coding theory [12] allows one to correct $\left\lfloor\frac{\Psi \boldsymbol{L}^{d_{H}\left(G_{\Lambda}\right)}}{2}\right\rfloor$ burst errors by uniform interleaving with depth $\Psi_{\boldsymbol{L}}$.

2. $G F\left(2^{q}\right)$ Symbol-Interleaving of $R S$ Codewords with $\Pi_{\Lambda^{\prime} \mid \Lambda^{\prime \prime}}$ : Now, by GF-symbol interleaving with depth $\Psi_{\boldsymbol{L}}$, a uniformlyinterleaved cyclic code over $\mathrm{GF}\left(2^{q}\right)$ can correct any single burst of $\Psi_{\boldsymbol{L}}((t-1) q+1)$ bit errors. This motivates the use of a $q$-bit symbol interleaver $\Pi_{\Lambda^{\prime} \mid \Lambda^{\prime \prime}}$ at the $\Lambda^{\prime} \mid \Lambda^{\prime \prime}$ partition.

3. $G F\left(2^{p}\right)$ Symbol-Interleaving of Cyclic Codewords with $\Pi_{\Lambda^{\prime \prime}}$ : For a $\mathrm{GF}\left(2^{p}\right)$ cyclic code used on $\left(\Lambda^{\prime \prime}+\Omega\right) \cap \mathcal{S}$, similar arguments can be drawn to motivate interleaving with $\Pi_{\Lambda^{\prime \prime}}$ on $p$-bit boundaries. If the single-burst correcting short codes of [11] are used, a uniform bit-interleaver over $G F(2)$ is sufficient. Since these are already constructed with interleaving to fit the coset codeword length (say, with depth $\Psi_{\Lambda^{\prime \prime}}$ ), the effective interleaver depth is $\Psi_{L} \Psi_{\Lambda^{\prime \prime}}$ 
Tab. 1. Combination of coding schemes investigated under realistic noise conditions

\begin{tabular}{|c|c|c|c|c|c|}
\hline Coset code & $R^{b}(\boldsymbol{L})$ & $\boldsymbol{G}_{\Lambda \mid \Lambda^{\prime}}$ & $\boldsymbol{G}_{\Lambda^{\prime} \mid \Lambda^{\prime \prime}}$ & $G_{\Lambda^{\prime \prime} \mid \Lambda^{\prime \prime \prime}}$ & $\Psi_{L}$ \\
\hline $\boldsymbol{L}_{1}$ & 47.7 & (3,6)-reg., [10] & $(6,32)-$ reg., rate-0.87 [4] & $(7,80)$-reg., rate-0.99 [4] & 1,24 \\
\hline $\boldsymbol{L}_{2}$ & 46.3 & & $(6,32)$-reg., rate-0.86 [4] & $(7,80)$-reg., rate-0.93 [4] & , \\
\hline $\boldsymbol{L}_{3}$ & 47.1 & $"$ & RS GF $\left(2^{8}\right)$, rate- 0.88 & RS GF $\left(2^{10}\right)$, rate- 0.95 & " \\
\hline $\boldsymbol{L}_{4}$ & 46.4 & " & RS GF $\left(2^{8}\right)$, rate- 0.88 & Single-Burst [11], rate- 0.93 & , \\
\hline
\end{tabular}

Due to the multi-level nature of the interleaver, notice from Fig. 1 that the decoded bits from one stage have to be re-interleaved in order to be used as the coset-labels of the next.

\section{Simulation Results with Colored Noise and Impulse Noise}

In this section, we present performance analysis of the proposed coding scheme under the conditions of ISI, colored noise and impulse noise. We augment the PAM-based scheme designed in Section III-C to withstand impulse noise. Our approach is to define a worst-case condition, and then design the coding and interleaving scheme accordingly to handle this case. This is clearly a suboptimal approach. Furthermore, even the worst-case scenario is a simplified assumption that does not always hold true, as will be explained further. However, to gauge the performance of the scheme, the results in impulse and Gaussian noise are compared to channel-capacity with only Gaussian noise. This provides a bound on the gap to the true channel-capacity in the presence of impulse noise.

Table 1 briefly outlines the combination of coding schemes that were investigated to this end. The channel under consideration is "channel 3". $R^{b}(\boldsymbol{L})$ refers to the final transmission rate after additional coding to handle bursts of impulse noise.

1) A "Worst-Case" Scenario: Consider the example of Section III-C, which operates at zero-excess bandwith at a baud-rate of 7.7 MHz. Since we have a binary lattice partition $\mathbb{Z}|2 \mathbb{Z}| 4 \mathbb{Z}$, each coset codeword in $\boldsymbol{L}$ consists of 2000 128-PAM symbols, as length of $\boldsymbol{G}_{\Lambda \mid \Lambda^{\prime}}=2000$. This implies a PAM symbol duration of $T_{s} \approx$ $0.13 \mu \mathrm{s}$ and a frame duration $T_{f}=2000 \cdot T_{s} \approx 260 \mu \mathrm{s}$. Consider the European electricity network and assume 6 synchronous noise impulses per $50 \mathrm{~Hz}$ AC cycle. To design for maximum number of burst errors during the cycle period, we assume the impulses are equally spaced in time. Then, an impulse event occurs every $3.3 \mathrm{~ms}$, or approximately every 12.7 coset codewords. We assume that the duration of each synchronous impulse noise burst is $t_{w, s} \approx 50 \mu \mathrm{s}$. Since the peak amplitudes of the synchronous bursts follow a Gaussian distribution $\mathcal{N}\left(0, G_{i} \sigma_{v}^{2}\right)$ in (2), we assume that, in the worst case, all symbols exposed to this burst would result in incorrectly decoded bits in the absence of coding. The synchronous burst spans, in the worst case, 385 PAM symbols.

Recall also that the duration of each asynchronous noise burst is assumed to be $t_{w, a}=100 \mu \mathrm{s}$, which corresponds to 770 PAM symbols. The average inter-arrival time of the asynchronous bursts is $\tau_{I A T, a}=100 \mathrm{~ms}$. Since the asynchronous impulses follow a Poisson arrival process, any number of asynchronous impulse bursts can arrive within a given interval. In particular, the probability of 2 or more such asynchronous impulses within a codeword interval is given by

$$
\begin{array}{r}
P\left\{\geq 2 \text { Poisson arrivals }, t=T_{f}\right\}= \\
1-e^{-\frac{t}{\tau_{I A T, a}}}\left(1+\frac{t}{\tau_{I A T, a}}\right) \approx 3.4 \times 10^{-6} .
\end{array}
$$

Consider an example of an interleaved scheme with $\Psi_{L}=24$, and hence $t=24 T_{f}$ sec's. Then, $P\{\geq 2$ Poisson arrivals $\} \approx$ $2 \times 10^{-5}$. When $\Psi_{L}=100$, the probability of 2 asynchronous arrivals is $\approx 3.3 \times 10^{-4}$. These probabilities are small, but of course, not negligible. For now, we assume that 2 asynchronous impulses will not occur within a $\Psi_{\boldsymbol{L}} T_{f}$ time interval; if it does,

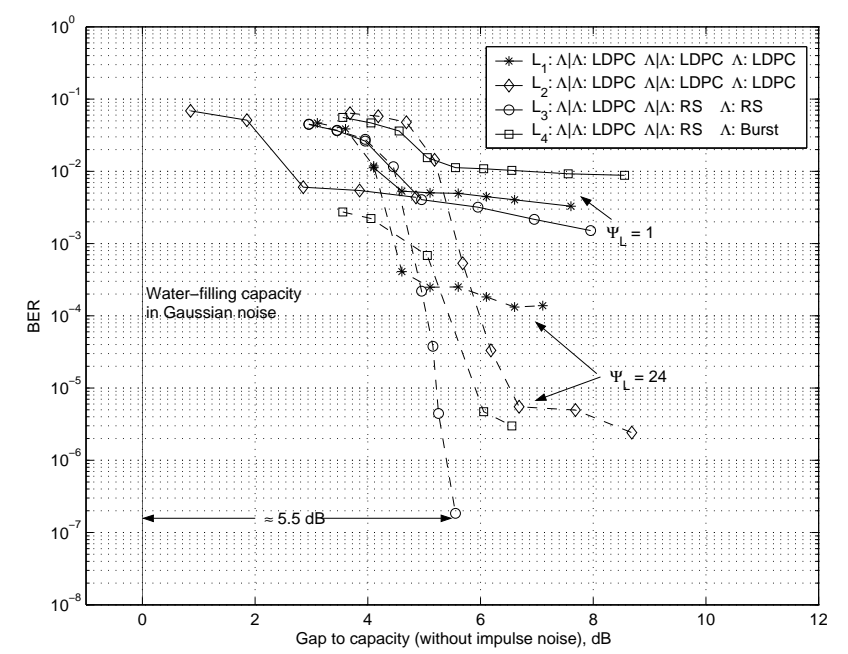

Fig. 4. Performance of coding schemes on "channel 3", in the presence of colored noise, residual ISI, synchronous and asynchronous impulse noise. Interleaver depth $\Psi=1$ and $\Psi=24$.

the error will be detected and corrected by a different means of error control discussed in Section IV-C.3.

Hence, the "worst-case" scenario can be stated as follows: one asynchronous impulse and a commensurate number of synchronous impulses occur within an interval $\Psi_{\boldsymbol{L}} T_{f}$ sec's. The burst-lengths $T_{w, s}$ and $T_{w, a}$ of the impulses are spanned completely within $\Psi_{L} T_{f}$. Let $l_{\Lambda \mid \Lambda^{\prime}}^{b}, l_{\Lambda^{\prime} \mid \Lambda^{\prime \prime}}^{b}$, and $l_{\Lambda^{\prime \prime}}^{b}$ denote the total phased-burst lengths, in bits, on $\Lambda\left|\Lambda, \Lambda^{\prime}\right| \Lambda^{\prime \prime}$, and $\Lambda^{\prime \prime}$ respectively. Let $l_{\Lambda}^{b}$ denote the burst length in terms of PAM symbols in $\Lambda$. Then,

$$
\begin{aligned}
l_{\Lambda}^{b} & =\underbrace{\left(\frac{\Psi}{t_{a r r, s} T_{f}} \cdot \frac{T_{w, s}}{T_{s}}\right)}_{\text {\# of synchronous impulses } \times \text { length }}+\underbrace{\frac{T_{w, a}}{T_{s}}}_{\text {asynchronous length }} \text { symbols, (9) } \\
l_{\Lambda \mid \Lambda^{\prime}}^{b} & =\log _{2}|\Lambda| \Lambda^{\prime} \mid \times l_{\Lambda}^{b}=l_{\Lambda}^{b} \text { bits, } \\
l_{\Lambda^{\prime} \mid \Lambda^{\prime \prime}}^{b} & =\log _{2}\left|\Lambda^{\prime}\right| \Lambda^{\prime \prime} \mid \times l_{\Lambda}^{b}=l_{\Lambda}^{b} \text { bits, } \\
l_{\Lambda^{\prime \prime}}^{b} & =\log _{2}\left|\left(\Lambda^{\prime \prime}+\Omega\right) \cap \mathcal{S}\right| \times l_{\Lambda}^{b}=5 l_{\Lambda}^{b} \text { bits }
\end{aligned}
$$

These burst-lengths are used in subsequent sections. We also use $\hat{l}_{\Lambda \mid \Lambda^{\prime}}^{b}, \hat{l}_{\Lambda^{\prime} \mid \Lambda^{\prime \prime}}^{b}$, and $\hat{l}_{\Lambda^{\prime \prime}}^{b}$ to denote the correctable total phased-burst lengths, in bits, on $\Lambda\left|\Lambda, \Lambda^{\prime}\right| \Lambda^{\prime \prime}$, and $\Lambda^{\prime \prime}$ respectively.

2) Trade-off Between Interleaver Depth and Burst-Error Coding: Our goal is to find a good combination of interleaving and burst-error correction needed to correct all burst-errors in the worst-case scenario. We first estimate the rates by which $\boldsymbol{G}_{\Lambda \mid \Lambda^{\prime}}, \boldsymbol{G}_{\Lambda^{\prime} \mid \Lambda^{\prime \prime}}$, and $\boldsymbol{G}_{\Lambda^{\prime \prime} \mid \Lambda^{\prime \prime \prime}}$ have their coding rates lowered to correct only the burst-errors. Computing this rate reduction can be very complex, since some codes can correct both random and burst errors simultaneously, and some errors due to Gaussian noise may overlap with burst errors. To simplify this analysis and to obtain just an estimate of the reduction in rate needed, we assume that all the code redundancy created by rate reduction is available for optimal phased-burst error correction. Then, the coding rates $R_{\Lambda \mid \Lambda^{\prime}}, R_{\Lambda^{\prime} \mid \Lambda^{\prime \prime}}$, and $R_{\Lambda^{\prime \prime}}$ are reduced by factors $\rho_{\Lambda \mid \Lambda^{\prime}}, \rho_{\Lambda^{\prime} \mid \Lambda^{\prime \prime}}$, 
and $\rho_{\Lambda} \prime \prime$ respectively, where

$$
\begin{aligned}
\rho_{\Lambda \mid \Lambda^{\prime}} & =2 \frac{l_{\Lambda \mid \Lambda^{\prime}}^{b}}{m \Psi_{\boldsymbol{L}}} \mathrm{b} / \mathrm{sym}, \quad \rho_{\Lambda^{\prime} \mid \Lambda^{\prime \prime}}=2 \frac{l_{\Lambda^{\prime} \mid \Lambda^{\prime \prime}}^{b}}{m \Psi_{\boldsymbol{L}}} \mathrm{b} / \mathrm{sym}, \\
\rho_{\Lambda^{\prime \prime}} & =2 \frac{l_{\Lambda^{\prime \prime}}^{b}}{5 m \Psi_{\boldsymbol{L}}} \mathrm{b} / \mathrm{sym} .
\end{aligned}
$$

The final rate is therefore

$$
\begin{aligned}
R^{b}(\boldsymbol{L})= & \frac{1}{T_{s}}\left[R_{\Lambda \mid \Lambda^{\prime}}\left(1-\rho_{\Lambda \mid \Lambda^{\prime}}\right)+R_{\Lambda^{\prime} \mid \Lambda^{\prime \prime}}\left(1-\rho_{\Lambda^{\prime} \mid \Lambda^{\prime \prime}}\right)+\right. \\
& \left.R_{\Lambda^{\prime \prime}}\left(1-\rho_{\Lambda^{\prime \prime}}\right)\right] \mathrm{bit} / \mathrm{sec} .
\end{aligned}
$$

Notice that the full Gaussian capacity cannot be reached, irrespective of $\Psi_{\boldsymbol{L}}$, since

$$
\begin{aligned}
\lim _{{ }^{\Psi} \rightarrow \infty} \rho_{\Lambda \mid \Lambda^{\prime}} & =\lim _{{ }^{\Psi} \boldsymbol{L}_{\rightarrow \infty}} \rho_{\Lambda^{\prime} \mid \Lambda^{\prime \prime}}=\lim _{{ }^{\Psi} \boldsymbol{L} \rightarrow \infty} \rho_{\Lambda^{\prime \prime}}, \\
& =\frac{1}{m} \frac{T_{f}}{t_{a r r, s}} \frac{T_{w, s}}{T_{s}} \mathrm{~b} / \mathrm{sym} .
\end{aligned}
$$

The limit is determined by the fraction of PAM symbols affected by synchronous noise, which is independent of $\Psi_{\boldsymbol{L}}$.

Suppose we are provided with a $2 \mathrm{dBmW}$ budget in transmit power, or corresponding SNR, for protection against burst noise. For the channel under consideration, this corresponds to 2.6 $\mathrm{Mbit} / \mathrm{sec}$ decrease in information rate to be used for impulse-noise protection. From the previous section, we estimate that $\Psi_{\boldsymbol{L}}=24$ is a good choice for error-free transmission at $R^{b}(\boldsymbol{L}) \approx 49.7-2.6$ $\mathrm{Mbit} / \mathrm{sec}$. With these rate and interleaver-depth estimates, our goal is to design a practical scheme with LDPC, RS, or burst-error correcting codes. Our design choice is to leave the LDPC code $\boldsymbol{G}_{\Lambda \mid \Lambda^{\prime}}$ unchanged since the code has been observed to perform well in the presence of impulse noise, even without reducing the rate. Table 1 shows the various component codes used in the simulations. To our knowledge, the LDPC codes selected from [4] are the best high-rate regular LDPC codes in AWGN channels.

3) Discussion of Simulation Results: The coset codes of Table 1 were tested under the channel and noise conditions described in earlier sections. All the coset codes, $\boldsymbol{L}_{1}, \ldots, \boldsymbol{L}_{4}$ use the same regular LDPC code from [10] for $G_{\Lambda \mid \Lambda^{\prime}}$. The comparative performance of the schemes with $\Psi_{\boldsymbol{L}}=1$ is shown in Fig. 4. The performance in all the figures is shown in terms of gap-to-capacity in Gaussian noise (since capacity in impulse-noise is difficult to compute). It can be seen that the impulse noise, coupled with lack of interleaving, has a devastating impact on BER performance for all schemes, irrespective of code type.

With interleaver depth of $\Psi_{\boldsymbol{L}}=24$, the BER performance is also shown in Fig. 4 . It can be seen that code $\boldsymbol{L}_{3}$ exhibits best performance, with no apparent error floor. The gap to capacity for rate $R^{b}\left(\boldsymbol{L}_{3}\right)$ is about $5.5 \mathrm{~dB}$ at a BER of $10^{-7}$. The code $\boldsymbol{L}_{4}$ also shows good performance until a BER of $3 \times 10^{-6}$, at which point an error floor is seen. The advantage of phased-burst error protection afforded by RS codes is evident. However, the complexity of a 1000 -symbol RS code over $\operatorname{GF}\left(2^{10}\right)$ is vastly higher than an interleaved $(195,182)$ binary cyclic code. The LDPC-only scheme $\boldsymbol{L}_{2}$ performs well until a BER $\approx 5 \times 10^{-6}$, but then exhibits an error floor. $\boldsymbol{L}_{1}$ still exhibits poor performance. This suggests that low-rate LDPC codes are more capable of handling burst errors.

Handling scenarios worse than the "worst-case": Although the simulations results show good performance with code $\boldsymbol{L}_{3}$ and $\Psi_{\boldsymbol{L}}=24$, conditions worse than the "worst-case" scenario of Section IV-C.1 occur with a probability $P_{b} \triangleq$ $P\left\{\geq 2\right.$ asynchronous impulse arrivals, $\left.t=24 T_{f}\right\} \approx 2 \times 10^{-5}$. To solve this anticipated error-floor, the proposed scheme can be combined with an automatic repeat-request (ARQ) protocol. Such schemes maintain coding rate - without sacrificing noise immunity - by using forward error-correction (FEC) until, say, probability of block error $<10^{-3}$. In the event of an uncorrectable error, a retransmit of the interleaved set of codewords is performed.

\section{CONCLUSion}

A simple LDPC-based coset coding scheme for power-line channels was investigated. The scheme combines LDPC and cyclic codes to achieve near-capacity performance in Gaussian noise, and to correct burst errors in impulse noise. At a BER of $10^{-11}$, the gap to un-shaped channel capacity is about $2 \mathrm{~dB}$ in Gaussian noise. To mitigate impulse noise, Reed-Solomon and burst error-correcting cyclic codes were investigated. An interleaving scheme is also proposed, consisting of distinct interleaving stages tailored to each level of the coset code. This results in increased immunity to burst noise caused by impulses. In the presence of colored Gaussian noise, synchronous and asynchronous impulse noise and residual ISI, the gap to channel-capacity of a Gaussian-noise-only channel is about $5.5 \mathrm{~dB}$ at a $\mathrm{BER} \approx 10^{-7}$.

\section{REFERENCES}

[1] M. Ardakani, T. Esmailian, and F.R. Kschischang. Near-capacity coding in multi-carrier modulation systems. IEEE Trans. Commun., 52(11):1880-1889, 2004.

[2] T.C. Banwell and S. Galli. On the symmetry of the power line channel. Proc. Int. Symp. Power-Lines Commun., pages 325-330, 2001.

[3] V. Degardin, M. Lienard, A. Zeddam, F. Gauthier, and P. Degauque. Classification and characterization of impulsive noise on indoor power lines used for data communications. IEEE Trans. Consum. Electronics, 48(4), Nov. 2002.

[4] I. Djurdjevic, J. Xu, A.-G. Khaled, and S. Lin. A class of Low-Density Parity-Check codes constructed based on Reed-Solomon codes with two information symbols. IEEE Commun. Lett., 7(7):317-319, July 2003.

[5] E. Eleftheriou and S. Ölçer. Low-Density Parity-Check codes for Digital Subscriber Lines. Proc. Intl. Conf. Commun., 3(28):17521757, Apr. 2002.

[6] G. D. Forney. Coset codes - Part I: Introduction and geometrical classification. IEEE Trans. Inform. Theory, 34(5):1123 - 1151, Sept. 1988.

[7] G. D. Forney, M.D. Trott, and S.-Y. Chung. Sphere-bound-achieving coset codes and multilevel coset codes. IEEE Trans. Inform. Theory, 46(3):820-850, May 2000.

[8] G.D. Forney and G. Ungerboeck. Modulation and coding for linear Gaussian channels. IEEE Trans. Inform. Theory., 44(6):2384-2415, Oct. 1998.

[9] J. Hou, P.H. Siegel, L.B. Milstein, and H.D. Pfister. Capacityapproaching bandwidth-efficient coded modulation schemes based on Low Density Parity-Check codes. IEEE Trans. Inform. Theory, 49(9):2141-2155, Sept. 2003.

[10] X.-Y. Hu, E. Eleftheriou, and D.M. Arnold. Regular and irregular progressive edge-growth Tanner graphs. IEEE Trans. Inform. Theory, 51(1):386-398, Jan. 2005.

[11] T. Kasami. Optimum shortened cyclic codes for burst-error correction. IEEE Trans. Inform. Theory., 9(2):105-109, Apr. 1963.

[12] S. Lin and D.J. Costello. Error control coding: fundamentals and applications. Pearson-Prentice Hall, Upper Saddle River, NJ, 2004.

[13] S. Rao, R. Hormis, and E. Krouk. The 4D PAM-8 proposal for 10G-Base-T. IEEE 802.3an 10G-Base-T Task Force, http://grouperieee.org/groups/802/3/tutorial/ nov03/rao_1_1103.pdf [Online], Nov. 2003

[14] T. Starr, J.M. Cioffi, and P.J. Silverman. Understanding Digital Subscriber Line Technology, Prentice Hall, Englewood Cliffs, NJ, 1999.

[15] G. Ungerboeck. 10G-Base-T Modulation and Coding, Set of Fixed Precoders, and Start-up. IEEE 802.3an 10G-Base-T Task Force, http://www.ieee802.org/3/an/public/nov04/ungerboeck_1_1104.pdf [Online], Nov. 2004.

[16] U. Wachsmann, R.F.H. Fischer, and J.B. Huber. Multilevel codes: Theoretical concepts and practical design rules. IEEE Trans. Inform. Theory, 45(5):1361-1391, Jul. 1999.

[17] M. Zimmermann and K. Dostert. Analysis and modeling of impulsive noise in broad-band powerline communications. IEEE Trans. Electromagnetic Compatibility, 44(1):249-258, Feb. 2002.

[18] M. Zimmermann and K. Dostert. A multipath model for the powerline channel. IEEE Trans. Commun., 50(4):553-559, Apr. 2002. 